\title{
Familienarmut - Bestandsaufnahme und Reformoption
}

Einkommensarmut von Familien bzw. von Kindern wird von allen politischen Parteien beklagt. Einschlägige Erfolge bei der Bekämpfung dieses gesellschaftlichen Problems bleiben jedoch aus. Der derzeit die Familienpolitik dominierende Ansatz, Kinderbetreuungsangebote auszubauen, um für Kinder aus einkommensarmen Familien den Zugang zu Bildung, zu sozialer Integration zu erleichtern, bringt nur begrenzte Verbesserungen. Zusätzliche Transferleistungen bleiben unerlässlich. Der 2005 eingeführte Kinderzuschlag zeigt wegen seiner restriktiven und komplizierten Ausgestaltung allerdings kaum Wirkung, sodass er in Richtung einer stärkeren Pauschalisierung der Anspruchsvoraussetzungen und verminderter Einkommensanrechnungen grundlegend reformiert werden müsste.

\section{Armutsgrenzen}

Familienarmut kann sich als Unterversorgungslage in verschiedenen Lebensbereichen niederschlagen. Der folgende Beitrag beschränkt sich allerdings auf Einkommensarmut (Abschnitt 1 und 2), ohne auf die Verschärfung des Problems im Falle der Kumulation von unzureichenden Versorgungslagen eingehen zu können. Im zweiten Teil des Beitrags wird ein Reformansatz erläutert (Abschnitt 3 und 4), der mit begrenztem fiskalischem Aufwand das Ausmaß von Familienarmut verringern könnte.

Zur Frage, bei welcher konkreten Einkommenshöhe Armut anzunehmen ist, wird es immer verschiedene Antworten geben, da Armut - ebenso wie Reichtum - ein normativer Begriff ist. Einigkeit besteht aber darüber, das es um ein relatives Konzept geht, das in irgendeiner Form auf das gesamtgesellschaftliche Wohlstandsniveau Bezug nimmt.

(1) Eine erste Abgrenzung von Armut orientiert sich an einem Einkommensmit- telwert als Synonym für den mittleren Lebensstandard einer Gesellschaft. Auf EUEbene hat man sich geeinigt, die Armutsgrenze bei $60 \%$ des nationalen Medians der bedarfsgewichteten Haushaltsnettoeinkommen zu setzen. Dabei erfolgt die Gewichtung der Haushaltseinkommen mit einer Äquivalenzskala, die den Bedarfszuwachs durch weitere Haushaltsmitglieder im Vergleich zur früheren Armutsmessung in Deutschland gering bewertet. Nach der sogenannten neuen OECD-Skala werden zweite und weitere Personen ab 14 Jahren mit dem Faktor 0,5, Kinder unter 14 Jahren mit 0,3 gewichtet, während die alternative alte OECD-Skala den Bedarf dieser Personen mit 0,7 bzw. 0,5 bewertet.

(2) Bei einem anderen Ansatz wird das gesetzlich verankerte soziokulturelle Existenzminimum, wie es für Deutschland im Sozialgesetzbuch (SGB II bzw. SGB XII) festgelegt ist, als Grenzwert herangezogen. Diese „politische“ Grenze ist teilweise individualisiert - insbesondere wegen der Berücksichtigung von Sonderbedarfen und der faktischen Wohnkosten neben dem Regelsatz von derzeit $347 €$ für einen Alleinstehenden. ${ }^{1} \mathrm{Zu}$ den von Armut nach ge-

Tabelle 1: Armuts- bzw. Bedürftigkeitsgrenzen nach Familientypen - in Euro per Monat -

\begin{tabular}{|c|c|c|c|c|}
\hline \multirow{3}{*}{ Familientyp } & \multicolumn{2}{|c|}{ Relative Armutsgrenze ${ }^{1}$} & \multicolumn{2}{|c|}{ Grundsicherung ${ }^{2}$} \\
\hline & Neue & Alte & Ohne & Mit maximalem \\
\hline & \multicolumn{2}{|c|}{ OECD-Skala } & \multicolumn{2}{|c|}{ Erwerbstätigen-Freibetrag } \\
\hline Alleinerziehend, 1 Kind $^{3}$ & 1.150 & 1.134 & 1.096 & 1.406 \\
\hline Paar, 1 Kind ${ }^{4}$ & 1.592 & 1.664 & 1.322 & 1.632 \\
\hline Paar, 2 Kinder ${ }^{4}$ & 1.858 & 2.042 & 1.600 & 1.910 \\
\hline \multicolumn{5}{|c|}{$\begin{array}{l}\text { 1) } 60 \text { \% des Medians der Nettoäquivalenzeinkommen; Berechnungen der Autorin auf Basis des SOEP } 2006 \text { (generiertes Monats- } \\
\text { einkommen). } \\
\text { 2) Regelleistung nach dem SGB II zuzüglich durchschnittlicher Wohnkosten von Hilfeempfängern (Bruttokaltmiete - vgl. Statisti- } \\
\text { sches Bundesamt 2006, Tabelle A2.6 - zuzüglich eines } 25 \% \text { igen Heizkostenzuschlags). In der neuen SGB II-Statistik fehlen bisher } \\
\text { entsprechende Angaben zu den Bruttoansprüchen. } \\
\text { 3) Kind unter } 7 \text { Jahren. } \\
\text { 4) Kind(er) unter } 14 \text { Jahren. }\end{array}$} \\
\hline Quelle: Berechnungen der Auto & & & & MITTEILUNGEN \\
\hline
\end{tabular}

○ WSI Mitteilungen 2002-2008

Diese Datei und ihr Inhalt sind urheberrechtlich geschützt. Nachdruck und Verwertung (gewerbliche Vervielfältigung, Aufnahme in elektronische Datenbanken, Veröffentlichung online oder offline) sind nicht gestattet. setzlicher Definition Betroffenen zählen neben den Empfängerinnen und Empfängern von Leistungen zur Sicherung des Existenzminimums - die sogenannte bekämpfte Armut - auch die Menschen in verdeckter Armut, die ihren Anspruch beispielsweise aus Unwissenheit oder Stigmatisierungsangst - nicht realisieren.

In Tabelle 1 sind die aus beiden Ansätzen resultierenden konkreten Armutsgrenzen für drei ausgewählte Familientypen dargestellt. Die relative Armutsgrenze von $60 \%$ des Medians ist sowohl bei Verwendung der neuen OECD-Skala als auch bei Annahme der alten OECD-Skala mit der höheren Bedarfsgewichtung berechnet worden. Daneben sind als Bedürftigkeitsgrenzen zum einen das Grundsicherungsniveau an sich und zum anderen der Grenzwert bei Ausschöpfung des maximalen Freibetrags bei Erwerbstätigkeit ausgewiesen, wobei durch-

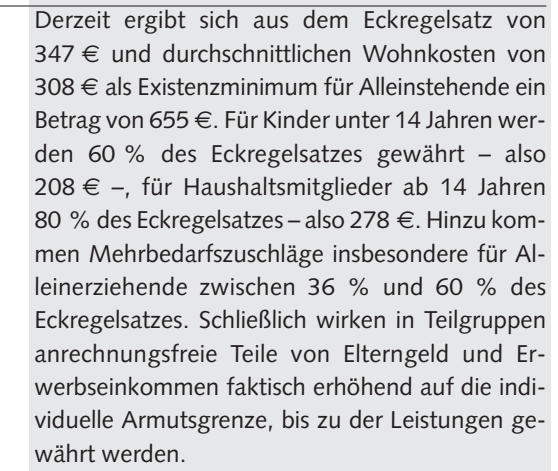
$347 €$ und durchschnittlichen Wohnkosten von $308 €$ als Existenzminimum für Alleinstehende ein Betrag von $655 €$. Für Kinder unter 14 Jahren werden $60 \%$ des Eckregelsatzes gewährt - also $208 €$-, für Haushaltsmitglieder ab 14 Jahren $80 \%$ des Eckregelsatzes - also $278 €$. Hinzu kommen Mehrbedarfszuschläge insbesondere für Alleinerziehende zwischen $36 \%$ und $60 \%$ des Eckregelsatzes. Schließlich wirken in Teilgruppen anrechnungsfreie Teile von Elterngeld und Erwerbseinkommen faktisch erhöhend auf die individuelle Armutsgrenze, bis zu der Leistungen gewährt werden.

Irene Becker, Dr. rer. pol., arbeitet als freiberufliche Wissenschaftlerin. Arbeitsschwerpunkte: Einkommensverteilung und soziale Sicherung. e-mail: I-H.Becker@t-online.de 
schnittliche Wohnkosten von Hilfeempfängern berücksichtigt wurden. Generell bezieht sich die Darstellung auf Familien mit Kindern unter 14 Jahren bzw. bei den Alleinerziehenden auf ein Kind unter 7 Jahren. Es zeigt sich, dass die gesetzliche Bedürftigkeitsgrenze bei Familien entgegen verbreiteten Annahmen nicht generell unter der empirisch abgeleiteten relativen Armutsgrenze liegt. Bei Alleinerziehenden fällt die gesetzliche Grenze im Falle von Erwerbstätigkeit sogar deutlich höher aus eine Folge des besonderen Mehrbedarfszuschlags in der Grundsicherung. Bei PaarFamilien ist das Grundsicherungsniveau an sich allerdings wesentlich niedriger als die relative Armutsgrenze. Je nachdem, wie hoch der Erwerbstätigenfreibetrag ausfällt, wird dieser Abstand aber vermindert. Der Vergleich der beiden relativen Armutsgrenzen schließlich zeigt, dass insbesondere bei mehreren Kindern die alte OECD-Skala mit ihren höheren Bedarfsgewichten zu einer vergleichsweise hohen Armutsgrenze führt - mit der Folge vergleichsweise hoher Armutsquoten (Becker/Hauser 2003, S. 183-187).

Je nach Grenzziehung fallen die Zahlen über Armut von Familien also unterschiedlich aus, und die Gruppe der Betroffenen unterhalb der gesetzlichen Armutsgrenze überschneidet sich nur teilweise mit der unter der relativen Armutsgrenze. Trotz der resultierenden Unschärfe des Bildes über das Ausmaß von Familienarmut zu einem Zeitpunkt zeigen alternativ gemessene zeitliche Entwicklungen aber ein ähnliches Muster; dies hat sich aus vorliegenden Untersuchungen ergeben (Hauser/Becker 2001). Somit gelten die folgenden Aussagen, die sich auf die relative Armutsgrenze mit Bezug zur neuen OECD-Skala beziehen, tendenziell auch für andere Abgrenzungen.

\section{Zur Entwicklung relativer Einkommensarmut in Deutschland}

Familienarmut ist keineswegs ein neues Phänomen, wie ein Blick auf die langfristige Entwicklung in Westdeutschland (Tabelle 2, oberer Teil) nach Ergebnissen der Einkommens- und Verbrauchsstichproben (EVS) zeigt. Bereits Anfang der 1970er Jahre lebte fast ein Fünftel der Alleinerziehen-

\begin{tabular}{|c|c|c|c|c|c|c|}
\hline & \multicolumn{6}{|c|}{ Westdeutschland (EVS)2 } \\
\hline & 1973 & 1978 & 1983 & 1988 & 1993 & 1998 \\
\hline Gesamtbevölkerung & 8,7 & 9,0 & 11,0 & 11,8 & 12,0 & 13,1 \\
\hline Ehepaare, 1 Kind & 2,7 & 3,5 & 5,8 & 6,5 & 6,7 & 11,8 \\
\hline Ehepaare, 2 Kinder & 5,0 & 5,3 & 6,6 & 6,9 & 9,2 & 8,8 \\
\hline Ehepaare, 3 u. m. Kinder & 9,3 & 12,7 & 8,3 & 9,6 & 16,6 & 10,4 \\
\hline Alleinerziehende, 1 Kind & $(18,0)$ & $(13,8)$ & 22,1 & 35,1 & 28,8 & 38,2 \\
\hline \multirow[t]{3}{*}{ Alleinerziehende, 2 u. m. Kinder } & $(17,1)$ & 34,9 & 38,2 & 47,3 & 47,1 & 41,9 \\
\hline & \multicolumn{6}{|c|}{ Gesamtdeutschland (SOEP) 3} \\
\hline & 1998 & 1999 & 2000 & 2001 & 2002 & 2003 \\
\hline Gesamtbevölkerung & 12,9 & 12,4 & 12,4 & 13,8 & 15,4 & 15,4 \\
\hline Paare, 1 Kind & $(12,0)$ & $(8,2)$ & $(7,2)$ & 10,4 & 12,1 & 13,6 \\
\hline Paare, 2 Kinder & $(12,0)$ & $(8,3)$ & $(10,1)$ & 11,0 & $(11,9)$ & $(10,1)$ \\
\hline Paare, 3 u. m. Kinder & $(18,5)$ & $(19,2)$ & $(13,3)$ & $(19,1)$ & $(25,0)$ & $(24,4)$ \\
\hline Alleinerziehende & $(40,7)$ & 49,4 & $(46,2)$ & 47,4 & 46,4 & 48,3 \\
\hline \multicolumn{7}{|c|}{$\begin{array}{l}\text { Werte in Klammern: Die Fallzahl liegt zwischen } 30 \text { und 100, sodass von einem erheblichen Fehlerspielraum ausgegangen werden } \\
\text { muss. } \\
\text { Als Kinder gelten alle Personen unter } 16 \text { Jahren sowie Personen von } 16 \text { bis } 24 \text { Jahren, sofern sie nicht erwerbstätig sind und } \\
\text { mindestens ein Elternteil im Haushalt lebt. } \\
\text { 1) } 60 \% \text { des Medians der Nettoäquivalenzeinkommen, neue OECD-Skala. } \\
\text { 2) Quelle: Hauser/Becker 2001, S. } 130 \text { f.; Datenbasis: Einkommens- und Verbrauchsstich- } \\
\text { proben (EVS). } \\
\text { 3) Quelle: Hauser/Becker 2005, S. 144; Datenbasis: Sozio-ökonomisches Panel (SOEP). }\end{array}$} \\
\hline
\end{tabular}

den und ihrer Kinder unterhalb der relativen Armutsgrenze. In den folgenden 25 Jahren hat sich die Quote aber etwa verdoppelt. Ein weniger dramatisches Bild ergibt sich für die Ehepaare mit Kindern. Zunächst waren hier insbesondere diejenigen mit drei und mehr Kindern betroffen; die Quoten der Paare mit weniger Kindern haben sich dem Niveau der Armutsquoten der „kinderreichen“"Ehepaare in den 1990er Jahren aber deutlich angenähert. Insgesamt sind die Armutsquoten nahezu aller Familientypen erheblich gestiegen, wobei das Niveau der Armutsquoten der Paar-Familien merklich höher - tendenziell überdurchschnittlich - ausfällt, wenn die alte OECD-Skala mit ihren höheren Bedarfsgewichten zugrunde gelegt wird (Hauser/Becker 2001, S. 128).

Für Gesamtdeutschland im Zeitraum 1998 bis 2003 ergibt sich eine ähnliche Situation, wie aus dem unteren Teil von $\mathrm{Ta}$ belle 2 - auf Basis einer anderen Datenquelle, des Sozio-ökonomischen Panels (SOEP), und trotz eines statistischen Unsicherheitsspielraums wegen geringer Fallzahlen - folgt. Etwa die Hälfte der Alleinerziehenden und ihrer Kinder und ein Viertel der Paare mit drei oder mehr Kindern lebten 2003 unterhalb der relativen Armutsgrenze, während Paare mit einem Kind oder zwei Kindern leicht unterdurchschnittlich betroffen waren.

Die Fortsetzung der auf dem SOEP basierenden Zeitreihe bis 2006 wird im Rahmen des dritten Armuts- und Reichtumsberichts veröffentlicht werden - voraussichtlich erst im Herbst 2008. Im Fol- genden werden daher eigene Berechnungsergebnisse auf Basis des SOEP 2006 vorgestellt. ${ }^{2}$ Diese beziehen sich auf Kinder, für die Kindergeld bezogen wurde und die in Kernfamilienhaushalten leben. Dabei ergibt sich eine Armutsquote von insgesamt $17,7 \%$ bei allerdings wesentlich höheren Betroffenheiten in einzelnen Teilgruppen: Bei einem Haushaltsvorstand ausländischer Staatsangehörigkeit liegt die Armutsquote der Kinder mit einem Drittel fast doppelt so hoch, in der Gruppe mit einem Haushaltsvorstand ohne beruflichen Ausbildungsabschluss leben zwei Fünftel der Kinder in Armut, und von den Kindern in Arbeitslosen- und sonstigen Nichterwerbstätigenhaushalten sogar sieben Zehntel.

Migrationshintergrund, Bildungsstand und Erwerbsstatus des Haushaltsvorstandes beeinflussen das Armutsrisiko von Kindern also gravierend. Dennoch wird die Sozialstruktur der Kinder in Einkommensarmut nicht von den "typischen“ Risikogruppen dominiert, da ihr Anteil an der Gesamtpopulation vergleichsweise klein ist. So leben etwa vier Fünftel der Kinder, deren Familien ein Einkommen unterhalb der $60 \%$-Armutsgrenze beziehen, mit einem deutschen Haushaltsvorstand, zwei Drittel leben mit einem Haushaltsvorstand, der eine berufliche Ausbildung oder gar ein Studium abgeschlossen hat, und

\footnotetext{
Sie sind allerdings wegen eines anderen Auswertungskonzepts (Becker/Hauser 2007) nicht vollkommen kompatibel mit der Zeitreihe bis 2003.
} 
immerhin ein Drittel lebt in einem Haushalt, in dem mindestens ein Elternteil in Vollzeit erwerbstätig ist oder beide Elternteile mindestens in Teilzeit (mehr als geringfügig) erwerbstätig sind.

Familienarmut im Sinne eines unzureichenden Einkommens hat also vielfältige Ursachen. Oft sind die Möglichkeiten von Eltern, einer Erwerbstätigkeit nachzugehen, nur eingeschränkt oder überhaupt nicht vorhanden - teilweise wegen fehlender Arbeitsnachfrage, teilweise wegen der Betreuungs- und Erziehungsaufgaben. Letzteres betrifft vorwiegend Frauen und ist insbesondere in den alten Bundesländern ein Problem, da hier das Angebot außerhäuslicher Kinderbetreuung noch viel $\mathrm{zu}$ gering ist. Wie aus der Strukturanalyse hervorgeht, ist Familienarmut aber nicht auf Arbeitslosen- und Teilzeiterwerbshaushalte begrenzt. Bei Niedriglöhnen reicht häufig auch eine Vollzeitbeschäftigung nicht für ein Überschreiten der Armutsschwelle. Und schließlich ist die Familiengröße mitentscheidend. Da Probleme häufig kumulieren, kann mit Maßnahmen, die nur an einzelnen Ursachen ansetzen - Ausbau der Kinderbetreuungsangebote, Kombilohn oder Mindestlohn -, Familienarmut nur teilweise bekämpft werden.

\section{Der bedarfsabhängige Kindergeldzuschlag - Eine Reformoption}

\section{1 DRINGENDER REFORMBEDARF}

Dem Problem der Familienarmut kann also nicht allein mit Maßnahmen zugunsten der Gruppen mit besonders hohen Armutsquoten begegnet werden. Es bedarf vielmehr auch eines allgemeinen, von der Armutsursache unabhängigen Konzepts. Die bisherigen familienpolitischen Maßnahmen greifen zu kurz. Dies ist insofern nicht verwunderlich, als das sächliche Existenzminimum eines Kindes in Höhe von derzeit $304 €$ zwar - neben einer Pauschale für den Betreuungs- und Erziehungsaufwand - von der Steuer freigestellt, nicht aber mit dem Kindergeld gewährleistet wird. Dementsprechend werden Familien im Niedrigeinkommensbereich, wenn das Haushaltseinkommen lediglich das elterliche Existenzminimum abdeckt, bedürftig im Sinne des SGB II.
Dem sollte mit dem Kinderzuschlag entgegengewirkt werden, der im Zuge der Hartz IV-Reform eingeführt worden ist. Dieser Kinderzuschlag wurde aber auf eine maximale Bezugsdauer von drei Jahren begrenzt und ist zudem so restriktiv und kompliziert ausgestaltet worden, dass er seine Wirkung weitgehend verfehlt hat. Dies ist im Wesentlichen auf die im Gesetz angelegte Mindesteinkommensgrenze in Höhe des Existenzminimums der Eltern bzw. des Elternteils zurückzuführen. Bei darunter liegenden Einkommen besteht kein Anspruch auf Kinderzuschlag, und die Familie wird auf die Grundsicherung nach dem SGB II verwiesen - die sie aber häufig nicht in Anspruch nimmt. Diese für Mindestsicherungsleistungen untypische untere Grenze ist zudem auf komplizierte Weise für jeden Einzelfall zu ermitteln, sodass Familien faktisch nicht abschätzen können, ob sie einen Anspruch auf Kinderzuschlag haben oder nicht. Hinzu kommt die hohe Transferentzugsrate von $70 \%$ bei Erwerbseinkünften bzw. $100 \%$ bei anderen Einkommensarten - z. B. bei Unterhaltszahlungen -, sodass der Einkommensbereich, in dem eine Anspruchsberechtigung gegeben ist, sehr schmal und die Zahl der potenziell begünstigten Kinder gering ist. Angesichts der institutionellen Ausgestaltung ist es nicht überraschend, dass bis Ende 2006830.000 Anträgen auf Kinderzuschlag nur gut 90.000 Bewilligungen gegenüberstanden und das Ausgabevolumen 2006 bei lediglich 140 Mio. $€$ lag.

\subsection{REFORMVORSCHLAG}

Für eine wirksame Bekämpfung von Familienarmut müsste der derzeitige Kinderzuschlag also grundlegend reformiert werden. Eine Möglichkeit besteht darin, ihn durch einen bedarfsabhängigen Kindergeldzuschlag zu ersetzen, der bei der Familienkasse zu beantragen wäre. ${ }^{3}$ Die Grundzüge des Vorschlags orientieren sich an Prinzipien des allgemeinen Familienleistungsausgleichs, wonach Familien unabhängig von der Ursache einer prekären Einkommenssituation und insbesondere ohne Bedingungen, die an die Grundsicherung für Arbeitsuchende geknüpft sind, zu fördern sind. Der Kindergeldzuschlag lässt sich wie folgt charakterisieren:

(1) Das Ziel, das Existenzminimum von Kindern im Rahmen des Familienleistungsausgleichs zu gewährleisten, erfordert die Aufstockung des Kindergeldes im Bedarfsfall auf die Höhe des sächlichen Existenzminimums, also um maximal $150 €$ auf $304 €$ - und zwar ohne zeitliche Befristung. Bei Alleinerziehenden sind allerdings verschiedene Mehrbedarfe anzunehmen, sodass hier der maximale Kindergeldzuschlag für das erste Kind um $100 €$ höher ausfallen sollte $(250 €)$.

(2) Das zu berücksichtigende Familieneinkommen umfasst alle vorrangigen Einkommen der Eltern bzw. des Elternteils und des Kindes mit Ausnahme des Kindergeldes.

(3) Leistungen nach dem Wohngeldgesetz und nach dem Bundesausbildungsförderungsgesetz sowie die Berufsausbildungsbeihilfe bleiben als nachrangige Transfers anrechnungsfrei; dementsprechend geht der Kindergeldzuschlag bei deren Bemessung als anzurechnendes Einkommen ein.

(4) Von dem zu berücksichtigenden Einkommen werden gegebenenfalls $300 €$ des Elterngeldes sowie ein Freibetrag in Höhe des pauschalisierten Existenzminimums der Eltern bzw. des Elternteils abgezogen. Es wird also keine akribische Einzelfallberechnung angestellt, sodass Familien viel leichter einschätzen können, ob sie anspruchsberechtigt sind oder nicht. Der Freibetrag sollte folgende Komponenten umfassen:

- den potenziellen Regelsatz bzw. die potenzielle Regelsatzsumme der (des) Eltern(teils) gemäß SGB II,

- einen Zuschlag von 50 \% des Eckregelsatzes zwecks pauschaler Berücksichtigung möglicher Freibeträge bei Erwerbstätigkeit nach $\$ 30$ SGB II und möglicher befristeter Zuschläge gemäß $\$ 24$ SGB II,

- und pauschalisierte Wohnkosten der (des) Eltern(teils) in Höhe der durchschnittlichen (warmen) Wohnkosten von Paaren ohne Kinder bzw. Alleinstehenden im Rahmen der Hilfe zum Lebensunterhalt am 01.01.2005, ${ }^{4}$ erhöht um einen Zuschlag

3 Dieser Vorschlag wurde im Rahmen eines von der Hans-Böckler-Stiftung geförderten Projekts entwickelt. Ausführliche Informationen finden sich in Becker/Hauser 2007.

4 Vgl. Deutsche Bundesregierung 2005, S. $106 \mathrm{der}$ Anhänge (Tabelle II.2). In diesem Zusammenhang muss auf die nicht mehr ganz aktuelle Sozialhilfestatistik zurückgegriffen werden, weil die seit der Hartz IV-Reform bestehende Grundsicherungsstatistik bisher nur Nettozahlungen, nicht aber die zugrunde liegenden Bruttobedarfe ausweist. 
von $5 \%$ insbesondere wegen gestiegener Energiepreise.

Daraus ergibt sich ein Freibetrag bei Paarhaushalten mit Kindern von $1.238 €$ und bei Alleinerziehenden von $860 €$.

(5) Das über den Freibetrag hinausgehende Einkommen soll nur zur Hälfte - statt wie bisher zu $70 \%$ bzw. $100 \%$ - auf den maximalen Kindergeldzuschlag angerechnet werden.

(6) Auf eine Berücksichtigung des Vermögens sollte verzichtet werden.

\subsection{WIRKUNGSWEISE}

Ein wesentlicher Unterschied des Reformkonzepts gegenüber dem derzeitigen Kinderzuschlag liegt in dem Verzicht auf eine Mindesteinkommensgrenze. Es bleibt den Eltern also unbenommen, den Kindergeldzuschlag in Anspruch zu nehmen, selbst wenn ihre Einkommensverhältnisse auf einen höheren ALG II-Anspruch schließen lassen, den sie aber nicht wahrnehmen - sei es aus Stigmatisierungsangst, aus Unwissenheit, weil sie den Verweis auf kleine Ersparnisse befürchten oder sich von dem bürokratischen Aufwand abschrecken lassen. Aus vorliegenden Schätzungen geht hervor, dass genau dieses die Ursachen für das große Ausmaß verdeckter Armut sind. Dem könnte durch einen vergleichsweise unbürokratischen Kindergeldzuschlag entgegengewirkt werden, insbesondere wenn der Leistungsträger, die Familienkasse, verpflichtet wird, bei sehr geringem Einkommen des Antragstellers diesen auf möglicherweise bestehende höhere ALG II-Ansprüche hinzuweisen.

Da der Kindergeldzuschlag stärker pauschalisierend als das ALG II bemessen wird, kann er in einem begrenzten Einkommensbereich je nach Lage des Einzelfalls $\mathrm{zu}$ einem etwas höheren oder etwas geringeren Familieneinkommen führen als die alternative Grundsicherung nach dem SGB II. Der Kindergeldzuschlag fällt tendenziell vergleichsweise hoch aus, wenn das Familieneinkommen nahe dem Existenzminimum laut SGB II liegt und überwiegend aus Unterhaltszahlungen oder Arbeitslosengeld I besteht - Einkommensarten, die nach dem SGB II voll angerechnet werden. Umgekehrt fällt in Erwerbstätigenhaushalten die Aufstockung durch ALG II und Sozialgeld tendenziell höher als der

Tabelle 3: Begünstigte und fiskalische Bruttokosten beim Reformkonzept des Kindergeldzuschlags

\begin{tabular}{|c|c|c|}
\hline & \multicolumn{2}{|c|}{ Restriktion bei Eigennutzern 1} \\
\hline & ohne & mit \\
\hline \multicolumn{3}{|l|}{ Begünstigte ... } \\
\hline \multicolumn{3}{|l|}{ - Kinder insgesamt } \\
\hline - in Mio. & 3.641 & 2.987 \\
\hline - in $\%$ von allen ${ }^{2}$ & 19,4 & 15,9 \\
\hline \multicolumn{3}{|l|}{ - Kinder von Alleinerziehenden } \\
\hline - in Mio. & 1.106 & 1.040 \\
\hline - in \% der Begünstigten & 30,4 & 34,8 \\
\hline - in \% der Kinder von Alleinerziehenden ${ }^{2}$ & 38,1 & 35,8 \\
\hline \multicolumn{3}{|l|}{ - Kinder in Paar-Familien } \\
\hline - in Mio. & 2.535 & 1.947 \\
\hline - in \% der Begünstigten & 69,6 & 65,2 \\
\hline - in \% der Kinder in Paar-Familien ${ }^{2}$ & 17,0 & 13,1 \\
\hline \multicolumn{3}{|l|}{ Ausgabenvolumen } \\
\hline - in Mrd. Euro p. a. & 4.548 & 3.706 \\
\hline - in \% der Kindergeldausgaben & 13,0 & 10,6 \\
\hline \multicolumn{3}{|c|}{$\begin{array}{l}\text { 1) Bei der restriktiven Variante wurde bei Familien in selbst genutztem Wohneigentum das anzurechnende Einkommen um eine } \\
\text { Pauschale für die ersparten Mietaufwendungen erhöht. Die Pauschale entspricht den (kalten) Kosten der Unterkunft (KdU), } \\
\text { die sich im Durchschnitt für die Empfänger von Hilfe zum Lebensunterhalt Ende } 2004 \text { ergeben haben (Alleinstehende: } 276 € \text {, } \\
\text { Ehepaare: } 355 € \text { E) zuzüglich der KdU, die bei der Berechnung des Existenzminimums von Kindern eingehen ( } 67 €) \text {. } \\
\text { 2) mit Kindergeldbezug. }\end{array}$} \\
\hline $\begin{array}{l}\text { Quelle: Becker/Hauser 2007, S. 21-31, auf Basis des SC } \\
\text { Generiertes Monatseinkommen. }\end{array}$ & & ITTEILUNGEN \\
\hline
\end{tabular}

Kindergeldzuschlag aus, da das Erwerbseinkommen nach dem SGB II nicht voll angerechnet wird. Weitere Abweichungen zwischen Einkommen mit aufstockendem ALG II und Einkommen nach Kindergeldzuschlag ergeben sich, wenn die Bandbreite individueller Wohnkosten - die für die Höhe des ALG II, nicht aber für die Höhe des Kindergeldzuschlags maßgeblich sindberücksichtigt wird. Diese Ergebnisunterschiede zwischen allgemeiner Grundsicherung einerseits und bedarfsabhängigem Kindergeldzuschlag andererseits sind die logische Konsequenz einer familienpolitischen Leistung mit lediglich pauschaler Bedürftigkeitsprüfung, die gerade durch den Verzicht auf stark einzelfallbezogene Prüfverfahren die Situation von Familien nahe der Armutsgrenze verbessern soll.

\section{1 \\ Erwartbare Reformeffekte}

Um die unmittelbaren Auswirkungen des skizzierten Kindergeldzuschlags abschätzen zu können, wurde auf Basis der Daten des SOEP 2006 eine Mikrosimulationsanalyse durchgeführt, ${ }^{5}$ und zwar für zwei Reformvarianten. Neben dem beschriebenen allgemeinen Konzept wurde auch eine restriktive Variante des Kindergeldzuschlags simuliert, bei der für Familien in selbst genutztem Wohneigentum das anzurechnen- de Einkommen um eine Pauschale für die eingesparte Miete erhöht wurde.

Bei Einführung des Kindergeldzuschlags würden etwa 3,6 Mio. Kinder bzw. 3 Mio. bei der restriktiven Variante begünstigt werden (Tabelle 3). Dies entspricht nahezu jedem fünften bzw. sechsten Kind, für das Kindergeld bezogen wird. Hier spiegelt sich die große Besetzungsdichte im unteren Einkommensbereich. Nach beiden Berechnungsvarianten würden zwar am häufigsten die in Paarhaushalten lebenden Kinder von der Reform profitieren. Sie wären zu zwei Dritteln die Begünstigten. Die Kinder von Alleinerziehenden sind aber überproportional vertreten: Für etwa jedes dritte Kind dieser Gruppe ergibt sich ein Anspruch auf Kindergeldzuschlag, während es unter den Kindern in Paarhaushalten ungefähr jedes sechste Kind, bei der restriktiven Variante jedes achte Kind ist.

Das potenzielle (Brutto-) Ausgabenvolumen ist im untersten Block von Tabelle 3 ausgewiesen. Demnach ist von 4,5 Mrd. $€$ bzw. 3,7 Mrd. € für das restriktive Modell auszugehen. Das entspricht ungefähr $13 \%$ bzw. $11 \%$ der derzeitigen Kindergeldaus-

\footnotetext{
Dabei wurde auf die für den Befragungsmonat erhobenen Einkommensarten Bezug genommen und unterstellt, dass die Finanzierung der Reform nicht zulasten der unteren Hälfte der Einkommensverteilung geht. Zu methodischen Details vgl. Becker/Hauser 2007, S. 21-31.
} 


\begin{tabular}{|c|c|c|c|}
\hline & \multirow[t]{2}{*}{$\begin{array}{l}\text { Vor } \\
\text { Reform }\end{array}$} & \multicolumn{2}{|c|}{$\begin{array}{c}\text { Nach Reform } \\
\text { Restriktion bei Eigennutzern }\end{array}$} \\
\hline & & ohne & mit \\
\hline \multicolumn{4}{|l|}{ Alleinerziehende } \\
\hline - insgesamt & 39,5 & 31,9 & 32,3 \\
\hline - mit 1 Kind & 34,3 & 27,0 & 27,6 \\
\hline - mit 2 u. m. Kindern & 45,0 & 37,2 & 37,4 \\
\hline \multicolumn{4}{|c|}{ Paar-Familien mit Kind(ern) } \\
\hline - insgesamt & 12,5 & 9,9 & 10,4 \\
\hline - mit 1 Kind & 12,7 & 11,5 & 11,6 \\
\hline - mit 2 Kindern & 9,5 & 7,8 & 8,5 \\
\hline - mit 3 u. m. Kindern & 18,6 & 11,6 & 12,3 \\
\hline Alle Familien & 16,1 & 12,8 & 13,3 \\
\hline \multicolumn{4}{|c|}{$\begin{array}{l}\text { 1) Nur Familien mit Kindern, für die Kindergeld bezogen wird. } \\
\text { 2) Armutsgrenze: } 60 \% \text { des Medians der Nettoäquivalenzeinkommen (neue OECD-Skala) } \\
\text { 3) Bei der restriktiven Variante wurde bei Familien in selbst genutztem Wohneigentum das anzurechnende Einkommen um } \\
\text { eine Pauschale für die ersparten Mietaufwendungen erhöht. Die Pauschale entspricht den (kalten) Kosten der Unterkunft (KdU), } \\
\text { die sich im Durchschnitt für die Empfänger von Hilfe zum Lebensunterhalt Ende } 2004 \text { ergeben haben (Alleinstehende: } 276 € \text {, } \\
\text { Ehepaare: } 355 € \text { ) zuzüglich der KdU, die bei der Berechnung des Existenzminimums von Kindern eingehen ( } 67 € \text { ). }\end{array}$} \\
\hline $\begin{array}{l}\text { Quelle: Becker/Hauser } 200 \\
\text { Generiertes Monatseinkom }\end{array}$ & des SOEP 2 & & EILUNGEN \\
\hline
\end{tabular}

gaben. Die Bruttokosten würden durch einige Einsparungen beim nachrangigen Wohngeld, bei ausbildungsbedingten Transfers sowie beim ALG II - sofern einige Anspruchsberechtigte den Bezug des Kindergeldzuschlags vorziehen - etwas vermindert werden.

Mit diesem begrenzten Mittelaufwand könnten die Armutsquoten von Familien erheblich reduziert werden. Besonders große Reformwirkungen zeigen sich bei den Alleinerziehenden. Wie aus Tabelle 4 hervorgeht, könnte die derzeit bei $40 \%$ liegende Armutsquote in dieser Gruppe um nahezu acht Prozentpunkte bzw. sieben Prozentpunkte bei der restriktiven Reformvariante reduziert werden. Trotz des vergleichsweise starken Effekts würde die Armutsquote der Alleinerziehenden und ihrer Kinder aber auch nach Einführung des Kindergeldzuschlags weiterhin bedrückend hoch ausfallen. Dies ist ganz überwiegend auf die große Zahl der Alleinerziehenden mit Bezug von ALG II und Sozialgeld bzw. Sozialhilfe zurückzuführen. Gut vier Fünftel der Alleinerziehenden und ihrer Kinder, die auch nach der Reform des Familienleistungsausgleichs unterhalb der Armutsgrenze verbleiben würden, beziehen eine Grundsicherungsleistung, deren Höhe offenbar hinter der relativen Armutsgrenze zurückbleibt. Wenn diese Teil-

gruppe ausgeklammert wird, erweist sich die Wirkung des Kindergeldzuschlags als wesentlich stärker, als es in Tabelle 4 ersichtlich wird. Nur bei knapp $6 \%$ der Alleinerziehenden ist die Armutslücke ${ }^{6}$ so groß - das Einkommen (ohne Kindergeld) bleibt also deutlich hinter dem pauschalisierten Existenzminimum des Elternteils $(860 €)$ zurück -, dass der Kindergeldzuschlag nicht zum Überschreiten der Armutsgrenze reicht. Aber selbst in diesen Fällen würde der neue Transfer eine Verbesserung der Situation bewirken, weil die Armutslücke zumindest deutlich vermindert wird; im Durchschnitt wird sie laut Simulation von $25 \%$ der Armutsgrenze auf weniger als die Hälfte (12 \%) bzw. um $319 €$ reduziert.

Bei den Paarfamilien zeigt sich ein geringerer Effekt des Kindergeldzuschlags, wenn die absolute Verminderung der Armutsquote (2,6 Prozentpunkte bzw. 2,1 Prozentpunkte bei der restriktiven Variante) betrachtet wird. Der relative Effekt ist aber auch hier erheblich - die Armutsquote von derzeit $12,5 \%$ würde um ein Fünftel zurückgehen. Dabei fällt die Reformwirkung umso größer aus, je mehr Kinder in der Familie leben. Bei den Familien, die trotz Einführung des Kindergeldzuschlags unter der relativen Armutsgrenze verbleiben, handelt es sich zu knapp der Hälfte um
Empfänger von nachrangigen allgemeinen Grundsicherungsleistungen. Bei der anderen Hälfte erreicht das um den Kindergeldzuschlag erhöhte Einkommen nicht die Armutsgrenze. Ihre materielle Situation würde sich dennoch erheblich verbessern, da die relative Armutslücke im Durchschnitt von $21 \%$ auf $14 \%$ zurückgehen würde; dies entspricht einer Einkommenserhöhung von durchschnittlich $267 €$. Wegen der hohen Erwerbsquote von einkommensarmen Elternpaaren würde sich mit dem Kindergeldzuschlag zwangläufig auch das Problem „Armut trotz Arbeit" verringern. Gut drei Viertel der potenziell Begünstigten in Paarfamilien leben in einem Erwerbstätigenhaushalt, und zwar mehrheitlich (zu wiederum drei Vierteln) in einem Vollerwerbshaushalt (Becker/Hauser 2007, S. 46).

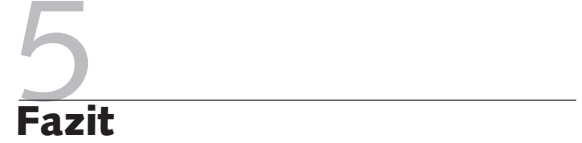

Der seit Jahrzehnten beobachtbaren Zunahme von Familienarmut könnte durch einen bedarfsabhängigen Kindergeldzuschlag begegnet werden. Bei begrenzten fiskalischen Belastungen von ca. 4 Mrd. $€$ p. a. würde die Armutsquote von Kindern von derzeit etwa $18 \%$ auf $14 \%$ zurückgehen, die der Familien von $16 \%$ auf $13 \%$. Die skizzierten positiven Effekte stellen sich allerdings nur bei einer hohen Inanspruchnahmequote ein. Diese ist bei einer familienpolitischen Leistung eher zu erreichen als beim leider mit vergleichsweise großer Stigmatisierung belegten ALG II/Sozialgeld. Durch eine vom Träger des Kindergeldzuschlags - der Kindergeldkasse - zu leistende informationelle Unterstützung von Familien mit weitergehendem Grundsicherungsanspruch könnte letztlich Familienarmut noch stärker reduziert werden, als es die Simulationsrechnung zeigt.

Als Armutslücke wird der Abstand des Haushaltsnettoeinkommens zur Armutsgrenze bezeichnet. 
Atkinson, A. B. (1998): Poverty in Europe, Oxford Becker, I./Hauser, R. (2003): Anatomie der Einkommensverteilung. Ergebnisse der Einkommens- und Verbrauchsstichproben 1969-1998. Forschung aus der Hans-Böckler-Stiftung 50, Berlin

Becker, I./Hauser, R. (2007): Vom Kinderzuschlag zum Kindergeldzuschlag: Ein Reformvorschlag zur Bekämpfung von Kinderarmut. Forschungsbericht an die Hans-Böckler-Stiftung, Riedstadt und Frankfurt am Main; auch erschienen als Arbeitspapier 5, Projekt "Soziale Gerechtigkeit" , Universität Frankfurt/Main

Deutsche Bundesregierung (2005): Lebenslagen in Deutschland. Der 2. Armuts- und Reichtumsbericht der Bundesregierung, Bonn Deutscher Bundestag (2007): Bericht über die Auswirkungen des $\S 6 a$ des Bundeskindergeldgesetzes (Kinderzuschlag) sowie über die gegebenenfalls notwendige Weiterentwicklung dieser Vorschrift. Unterrichtung durch die Bundesregierung, BT-Drucksache 16/4670
Esping-Andersen, G. (2004): Die gute Gesellschaft und der neue Wohlfahrtsstaat, in: Zeitschrift für Sozialreform 1-2, S. 189-210 Hauser, R./Becker, I. (2001): Einkommensverteilung im Querschnitt und im Zeitverlauf 1973-1998. Studie zum Ersten Armuts- und Reichtumsbericht der Bundesregierung im Auftrag des Bundesministeriums für Arbeit und Sozialordnung (jetzt: BMAS), Reihe Lebenslagen in Deutschland, Bonn

Hauser, R./Becker, I. (2005) unter Mitarbeit von Krause, P./Grabka, M./Mattil, B./Kortmann, K.: Verteilung der Einkommen 1999-2003. Studie zum Zweiten Armuts- und Reichtumsbericht der Bundesregierung im Auftrag des Bundesministeriums für Gesundheit und Soziale Sicherung (jetzt: BMAS), Reihe Lebenslagen in Deutschland, Bonn Statistisches Bundesamt (2006): Fachserie 13, Sozialleistungen, Reihe 2.1, Sozialhilfe 2004, Stuttgart 